\title{
Covertly active and progressing neurochemical abnormalities in suppressed HIV infection
}

Lucette A. Cysique, PhD, Lauriane Jugé, PhD, * Thomas Gates, MA (Clin Neuropsychol), * Michael Tobia, BA Sc (Hons), Kirsten Moffat, BA AppSc (MRT), Bruce J. Brew, MBBS (Hons), MD, FRACP, FAAN, $\dagger$

and Caroline Rae, PhD†

Neurol Neuroimmunol Neuroinflamm 2018;5:e430. doi:10.1212/NXI.0000000000000430

\section{Abstract}

\section{Objective}

To assess whether HIV-related brain injury is progressive in persons with suppressed HIV infection.

\section{Methods}

Seventy-three HIV+ virally suppressed men and 35 HIV- men, screened for psychiatric and alcohol/drug use disorders, underwent neuropsychological evaluation and proton magnetic resonance spectroscopy $\left({ }^{1} \mathrm{H}-\mathrm{MRS}\right)$ at baseline and after and $23 \pm 5$ months. ${ }^{1} \mathrm{H}-\mathrm{MRS}$ included brain regions known to be vulnerable to HIV and aging: frontal white matter (FWM), posterior cingulate cortex (PCC), and caudate area (CA). Major brain metabolites such as creatine (Cr: marker of cellular energy), $\mathrm{N}$-acetyl aspartate (NAA: marker of neuronal integrity), choline (marker of cellular membrane turnover), glutamate/glutamine (excitatory/inhibitory neurotransmitter), and myo-Inositol (mI: marker of neuroinflammation) were calculated with reference to water signal. Neurocognitive decline was corrected for practice effect and baseline HIV-associated neurocognitive disorder (HAND) status.

\section{Results}

Across the study period, $44 \%$ had intact cognition, $42 \%$ stable HAND (including the single case that improved), $10 \%$ progressing HAND, and $4 \%$ incident HAND. When analyzing the neurochemical data per neurocognitive trajectories, we found decreasing PCC $\mathrm{Cr}$ in all subgroups compared with controls $(p<0.002)$. In addition, relative to the HIV- group, stable HAND showed decreasing FWM $\mathrm{Cr}$, incident HAND showed steep FWM Cr reduction, whereas progressing HAND had a sharply decreasing PCC NAA and reduced but stable CA NAA. When analyzing the neurochemical data at the group level (HIV+ vs HIV-groups), we found stable abnormal metabolite concentrations over the study period: decreased FWM and PCC Cr (both $p<0.001$ ), decreased PCC NAA and CA NAA (both $p<0.05$ ) and PCC mI increase $(p<0.05)$. HIV duration and historical HAND had modest effects on metabolite changes.

\section{Conclusions}

Our study reveals covertly active or progressing HIV-related brain injury in the majority of this virally suppressed cohort, reflecting ongoing neuropathogenic processes that are only partially worsened by historical HAND and HIV duration. Longer-term studies will be important for determining the prognosis of these slowly evolving neurochemical abnormalities.
Correspondence

Dr. Cysique:

Icysique@unsw.edu.au 


\section{Glossary}

${ }^{1} \mathrm{H}$-MRS = proton magnetic resonance spectroscopy; ANI = asymptomatic neurocognitive impairment; $\mathbf{C A}=$ caudate area; cART = combination antiretroviral; $\mathbf{C r}=$ creatine; $\mathbf{C h o}=$ choline; $\mathbf{F W M}=$ frontal white matter; $\mathbf{G D S}=$ Global Deficit Score; $\mathbf{G l x}=$ glutamate/glutamine; $\mathbf{G M}=$ gray matter; HAND = HIV-associated neurocognitive disorder; $\mathbf{m I}=$ myo-Inositol; $\mathbf{M N D}=$ mild neurocognitive disorder; NAA $=\mathrm{N}$-acetyl aspartate; $\mathbf{P C C}=$ posterior cingulate cortex .

The existence of neurochemical abnormalities assessed by proton magnetic resonance spectroscopy $\left({ }^{1} \mathrm{H}-\mathrm{MRS}\right)$ in vivo has been robustly demonstrated in the HIV-infected (HIV+) population across many cross-sectional studies. ${ }^{1,2}$ However, longitudinal prognostic studies are few.

Indeed the long-term study of neurochemical abnormalities by ${ }^{1} \mathrm{H}-\mathrm{MRS}$ may have several prognostic benefits: (1) It may inform whether virally suppressed HIV+ persons with stable cognition or stable HIV-associated neurocognitive disorder (HAND) across several years (the most prevalent neurocognitive trajectories in chronic treated HIV infection ${ }^{3,4}$ ) demonstrate active HIV-related brain injury rather than a "burnt out" form of injury reflecting irreversible historical damage. (2) It may assist in the early detection of HAND incidence and progression, despite viral suppression. (3) The profile of neurochemical changes can also provide unique insights into the underlying neuropathogenic processes, and whether these are fundamentally different from HIV replication-driven neuropathogenesis.

To the best of our knowledge, only 1 longitudinal ${ }^{1} \mathrm{H}-\mathrm{MRS}$ study has been conducted in chronic treated HIV+ patients. ${ }^{5}$ This study found a reduction of all metabolites measured across the 2-year study period including $\mathrm{N}$-acetyl aspartate (NAA) and choline (Cho) in the frontal white matter (FWM); NAA, creatine (Cr), Cho, and glutamate/glutamine (Glx) in the midfrontal cortex, and Glx reduction in the basal ganglia. Limitations of this study included the absence of a control sample and that not all HIV+ participants were virally suppressed. Both aspects are critical to determine whether such neurochemical changes have clinical relevance or are just an epiphenomenon of chronic HIV infection without neuropathogenic importance.

\section{Methods}

The major aim of this study was to assess whether HIV-related brain injury follows a progressive trajectory in persons with suppressed HIV infection ${ }^{6}$ :

1. By characterizing the pattern of neurochemical changes in men with chronic and treated HIV infection relative to an age-comparable HIV- control group over a $23 \pm 5$ month-period;

2. By determining whether clinically relevant neurocognitive trajectories are associated with distinct longitudinal neurochemical profiles;
3. By measuring the effects of age, age $\times$ HIV interaction, HIV disease markers, and treatment on neurochemical changes.

We hypothesized that HAND in the context of viral suppression primarily indicates active evolving brain injury. We predicted that, relative to HIV- controls, HIV+ participants would show a significant longitudinal reduction in NAA in all studied brain regions and a longitudinal increase in FWM myo-inositol $(\mathrm{mI})$. We also predicted that these changes would occur in a manner mostly independent of the past immunocompromise and HIV duration.

\section{Participants}

The current investigation is based on the Australian HIV and Brain Aging Research Program, a prospective study investigating the effects of HIV infection in the brains of middle-aged HIV+ and HIV- persons (i.e., aged 45+). Study participants' baseline characteristics have been published previously ${ }^{7}$ and are summarized in the legend of table e-1 at links.lww.com/NXI/A24.

At baseline, $84 \mathrm{HIV}+$ men and $42 \mathrm{HIV}$ - men completed an MRI/MRS scan, a standard neuropsychological evaluation, and a laboratory examination. Seventy-three HIV+ and 35 HIV- men returned for follow-up assessment designed to be approximately 18 months later (test-retest interval: mean = 23 months, SD $=5$ months). HIV+ participants lost to follow-up $(\mathrm{N}=11)$ did not statistically differ from those who completed the study on most baseline demographic characteristics and disease factors (table e-1), including the overall level of neurocognitive impairment at baseline. However, HIV+ patients who did not complete their followup assessment were significantly more likely to have reached a detectable viral load level $(>50$ copies $/ \mathrm{mL}$ ) during the study period compared with HIV+ patients who completed the study (lost to follow-up $=45 \%$, vs completed $=8 \%$; $p<$ $0.0004)$. In the HIV- group, cases who were lost to followup $(\mathrm{N}=7)$ tended to have higher verbal IQ and slightly more depressive symptoms than HIV- cases who completed the study (both $p=0.05$ ).

\section{Procedures and investigations}

\section{Neuropsychological evaluation}

The standard neuropsychological evaluation has been described previously by Cysique et al. ${ }^{8}$ and is outlined more in detail below. It was completed within an average of a month of the MRI/MRS scan. 


\section{${ }^{1} \mathrm{H}-M R S$ acquisition and analysis}

The detailed ${ }^{1} \mathrm{H}$-MRS protocol has been reported by Cysique et al. ${ }^{7}$ Quantitative analysis of spectra was completed using jMRUI (Version 3.0 [http://www.mrui.uab.es/] and the AMARES algorithm. ${ }^{9}$ The following signals ${ }^{10}$ were fitted and expressed as ratios with respect to the unsuppressed water signal $\left(\mathrm{H}_{2} \mathrm{O}\right)$ : NAA (traditionally interpreted as a marker of neuronal density/loss), Cho (marker of cellular membrane turnover), $\mathrm{Cr}$ (marker of cellular energy), Glx (the 2 first peaks were retained mostly representing the major excitatory neurotransmitter ${ }^{11}$ ), and $\mathrm{mI}$ (marker of neuroinflammation in the context of $\mathrm{HIV}^{5}$ ). The longitudinal reliability of the ${ }^{1} \mathrm{H}$-MRS voxel placement by an expert operator (following guidelines reported by Cysique et al. ${ }^{7}$ was tested by comparing the fraction of gray matter [GM],WM, and CSF within the study voxels to the reference $\mathrm{H}_{2} \mathrm{O}$ signal. GM, WM, and CSF volumes were computed using partial volume code for Philips MRS data (Nia Goulden and Paul Mullins, Bangor Imaging $\mathrm{Unit}^{12}$ ). There were no time differences in volume distributions between the HIV+ and HIV- groups or as functions of their neurocognitive trajectories. Moreover, across the study period, the reference $\mathrm{H}_{2} \mathrm{O}$ signal did not significantly change as a function of HIV status (interaction term HIV status $\times$ time, $p=0.10$; Std $\beta=-0.12$ ). However, the $\mathrm{H}_{2} \mathrm{O}$ signal was different as a function of neurocognitive trajectories. We found that those with progressing and incident HAND had significantly increased $\mathrm{H}_{2} \mathrm{O}$ signals in all voxels compared with all other groups (FWM: $p<0.004$; posterior cingulate cortex $[\mathrm{PCC}]: p=0.05$; caudate area $[\mathrm{CA}]: p=0.03)$. Therefore, this was accounted for in the statistical analyses to maintain $\mathrm{H}_{2} \mathrm{O}$ as an adequate metabolite signal reference.

\section{Definition of neurocognitive trajectories}

Detailed testing procedures have been published previously. ${ }^{8,13}$ Briefly, the Australian HIV and Brain Aging Research Program uses a neuropsychological test battery that is in use in international neuroHIV research. ${ }^{8,13}$ Neuropsychological impairment was defined with the standard Global Deficit Score (GDS) method $^{14}(\mathrm{GDS} \geq 0.5){ }^{15}$

Clinically meaningful cognitive change (improve and decline) vs stability was defined using published norms for change (see reference 3 for further details). To determine the neurocognitive trajectories, we combined the participants' baseline impairment classification with their neurocognitive change status at followup. This produced 4 categories (figure 1): (1) stable intact: cases who had normal neuropsychological performance at baseline and remained stable at follow-up, (2) stable HAND: cases who had HAND at baseline and remained stable at follow-up, (3) HAND progressing: cases who had HAND at baseline and declined significantly at follow-up, and (4) incident HAND: cases who had normal neuropsychological performance at baseline and significantly declined at follow-up. We note that the incident HAND cases also had impaired performance corrected for practice effect (2 asymptomatic neurocognitive impairment [ANI] and 1 mild neurocognitive disorder [MND]) on the
Figure 1 Neurocognitive trajectories' prevalence

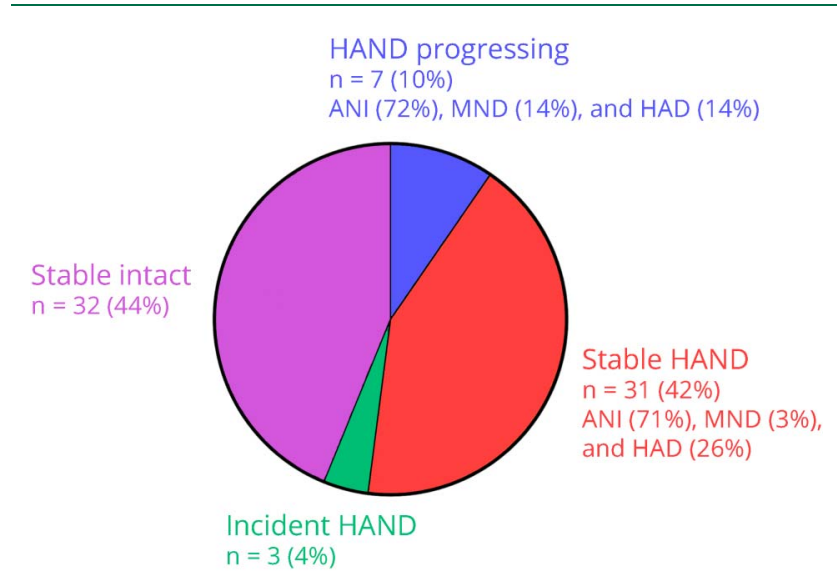

The largest subgroups represented HIV+ individuals who remained cognitively intact across the study period (stable intact; 44\%) and those who had baseline HIV-associated neurocognitive disorder (HAND) and remained stable (stable HAND; 42\%). Clinically meaningful cognitive decline was detected in a minority, and we distinguished those who had progressing HAND from those who had incident HAND. Note that all incident cases reached impaired performance level at the follow-up (2 ANI and 1 MND). $\mathrm{ANI}=$ asymptomatic neurocognitive impairment; HAD = HIV-associated dementia; MND = mild neurocognitive disorder.

follow-up testing. Only 1 case significantly improved and was thus grouped with the "Stable Intact" group.

\section{Ethics}

All individuals provided written informed consent before participating in the study, and the protocol was approved by the St. Vincent's Hospital and the University of New South Wales Human Research Ethics Committees.

\section{Statistical analysis}

A significant change in brain metabolites was determined using linear regression with a time effect, a group effect, and a time $\times$ group interaction effect. The age effect was examined using linear regression with main effects of age, time and group, as well as age $\times$ time interaction, age $\times$ group interaction, and age $\times$ time $\times$ group 3 -way interaction terms. All models were adjusted for percentage WM volume or percentage GM volumes in each relevant voxel.

To explore the profiles of brain metabolite concentrations across the 4 predefined neurocognitive trajectories in the HIV+ group and the HIV- group, we used linear regression models with a time effect, a neurocognitive trajectory effect, and the interaction between those 2 terms. Post hoc Dunnett comparison was conducted with the HIV-control follow-up as the reference term to better delineate the profile of each subgroup.

To determine whether baseline HIV disease markers and treatment factors were predictive and/or associated with brain metabolite changes over time in the HIV+ group, we conducted linear regression analyses with the metabolites that were found to be significantly different between the overall 
$\mathrm{HIV}+$ and HIV - groups as the outcomes to reduce the number of experiment-wise comparisons and concentrate on clinically meaningful effects. The following predictors were assessed for their correlations (if $r \geq 0.30$, a redundant predictor was removed to reduce the number of relevant variables in the final regression model): baseline HIV duration (years), nadir CD4 T-cell count (copies/mL), total number of AIDSdefining illnesses (other than HAND), history of HAND (yes/no; dummy coded, see reference 3 for further details), baseline combination antiretroviral (cART) duration (months), baseline CNS penetration effectiveness rank score, ${ }^{16}$ difference in CD4 T-cell count between baseline and follow-up, cART change during the study period (yes/no; dummy coded), and viral blip during the study period $(<50$ copies/mL, undetectable; yes/no; dummy coded). Nadir CD 4 count and total number of AIDS-defining illnesses were associated $(r=-0.45 ; p<0.0001)$; thus, nadir CD4 was retained. cART change during the study period and viral blip were associated $(r=0.30 p=0.01)$; thus, viral blip was retained.

Statistical analyses were conducted in JMP version 12 (SAS, Inc, Cary, NC). Statistical significance was set at $\alpha<0.05$, whereas effect sizes were produced for all analyses to aid interpretation.

\section{Results}

\section{Longitudinal brain metabolite changes}

Figure 2 presents metabolite changes over time as a function of neurocognitive trajectories and in the HIV- sample. In addition, the change in metabolite profile for each neurocognitive trajectory is summarized in table 1 .

Figure 2 Neurochemical profiles for each neurocognitive trajectory

A. FWM $\mathrm{Cr}$ and neurocognitive trajectories

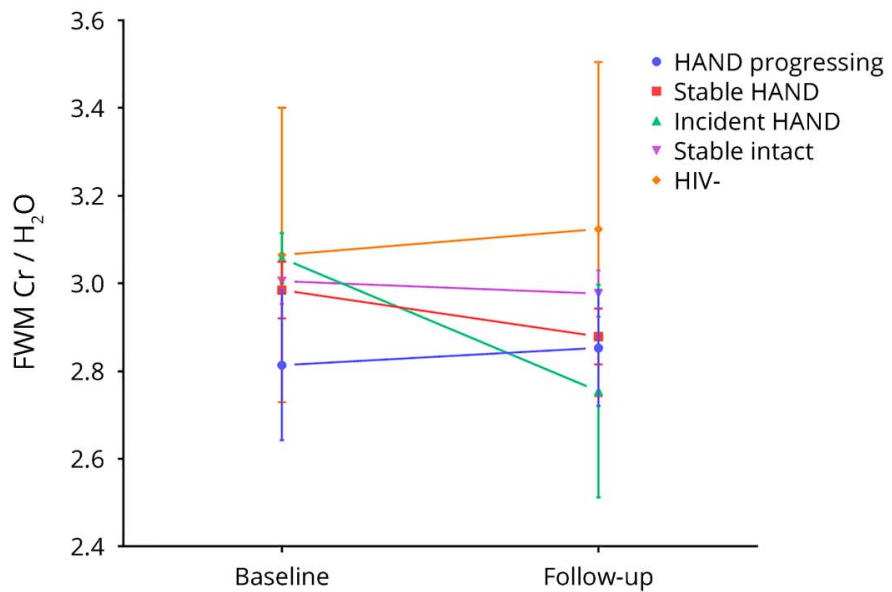

C. PCC $\mathrm{Cr}$ and neurocognitive trajectories

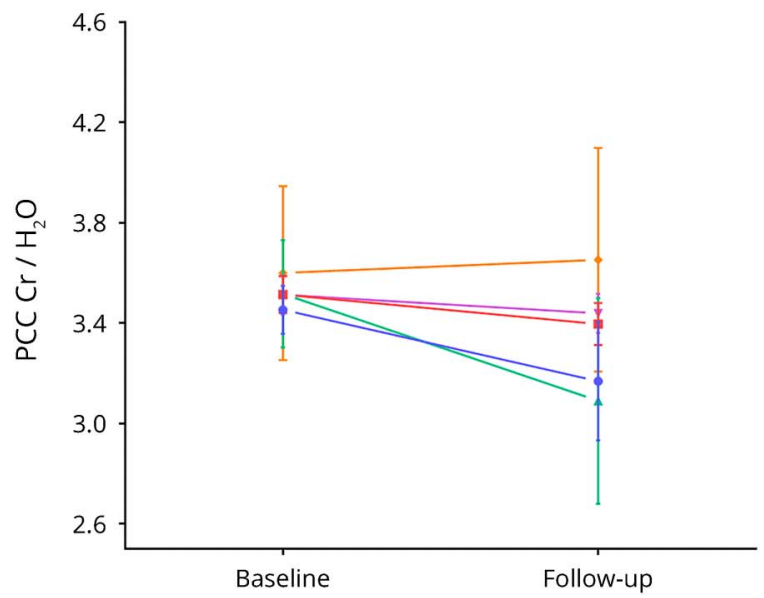

B. PCC NAA and neurocognitive trajectories

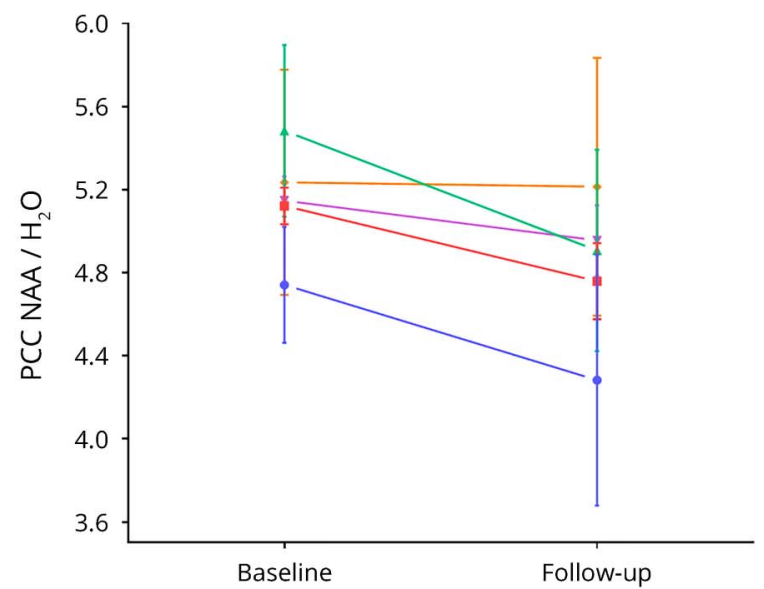

D. CA NAA and neurocognitive trajectories

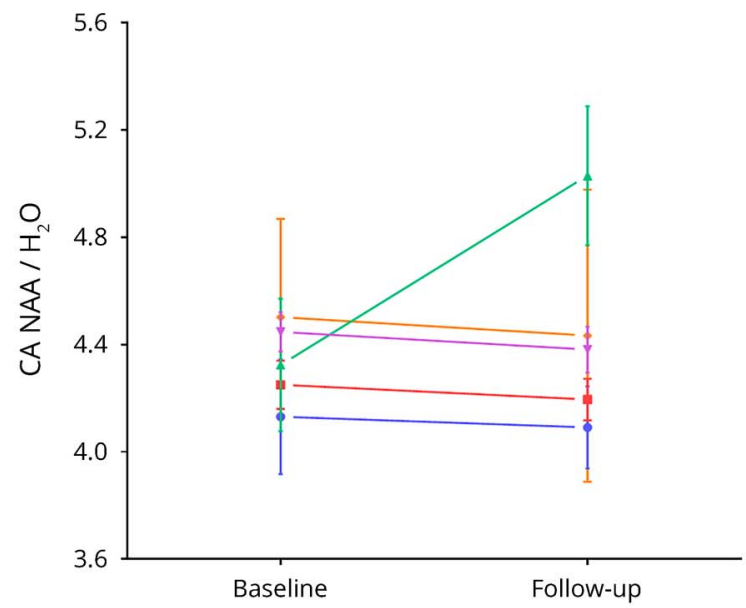

(A) Frontal white matter creatine (FWM Cr); (B) PCC N-acetyl aspartate (PCC NAA); (C) PCC Cr; (D) caudate area NAA (CA NAA). HAND = HIV-associated neurocognitive disorder; $\mathrm{PCC} \mathrm{Cr}=$ posterior cingulate cortex $\mathrm{Cr}$. 
Neurocognitive trajectories had an overall effect on FWM Cr concentration compared with the HIV- sample ( $p=0.0007$; Std $\beta=0.37$ ) (figure $2 \mathrm{~A}$ ). More specifically, Dunnett comparisons using the HIV- control follow-up as the reference showed that the stable HAND cases had lower $\mathrm{Cr}$ at follow-up $(p=0.004)$. The incident HAND group demonstrated a sharp decline in moderate size (time $\times$ incident cases: $p=0.29$; $\operatorname{Std} \beta$ $=0.19$ ), although this was not statistically significant, probably because of the small sample size (post hoc: $p=0.07$ ).

PCC NAA was reduced across the study period, particularly in cases with progressing HAND $(p<0.008$; Std $\beta=-0.34)$ (figure $2 \mathrm{~B}$ ), and further confirmed by post hoc Dunnett comparisons (compared with HIV- control follow-up: $p=0.004)$. In addition, the post hoc comparisons showed that the stable HAND cases had lower NAA at follow-up compared with HIV- controls $(p<0.02)$.

Neurocognitive trajectories had an overall effect on PCC Cr concentration compared with the HIV- group ( $p<0.002$; Std $\beta=0.34$ ). Post hoc Dunnett comparisons were different for all HIV+ subgroups relative to HIV- controls at follow-up (stable intact: $p<0.05$; stable HAND: $p<0.02$; incident HAND: $p<0.04$; and particularly for the progressing HAND $[p<0.008]$ ) (figure 2C).

CA NAA was moderately reduced across the study period in cases with progressing HAND $(p<0.02$; Std $\beta=-0.31)$ and stable HAND $(p<0.02$; Std $\beta=-0.26)$. Moreover, cases with incident HAND had moderately low NAA at baseline $(p<$ 0.05 ; Std $\beta=0.33$ ), which sharply increased over the study period $(p<0.05$; Std $\beta=-0.37$ ) (figure 2D). Post doc Dunnett comparisons confirmed those effects for incident HAND ( $p=$ 0.03 ) and showed that stable HAND had lower NAA compared with HIV- controls at follow-up $(p<0.04)$.

When restricting the analyses to cases with ANI at baseline by excluding only the MND cases, the overall effect of the neurocognitive trajectories remained significant on the FWM Cr, PCC NAA, PCC Cr, and CA NAA but with smaller effect sizes. When restricting the analyses to cases with MND at baseline by excluding only the ANI cases, the overall effect of the neurocognitive trajectories increased to large effect sizes. Because some subgroups were very small, these analyses are only indicative.

When grouping the HIV+ sample relative to HIV- controls, we found that across the study period, the FWM $\mathrm{Cr}$ $(p<0.001)$, PCC Cr $(p<0.01)$, PCC NAA $(p<0.05)$, and CA NAA $(p<0.05)$ were reduced; whereas the PCC $\mathrm{mI}$ $(p<0.05)$ was elevated (table 2). There was no change in these findings when age and associated interaction terms were added to the models as covariates.

\section{HIV disease markers and treatment factors}

The mean CD4 T-cell count increased during the study period from 559 copies $/ \mathrm{mL}$ at baseline to 654 copies $/ \mathrm{mL}$ at follow-up $(p<0.0001)$, and no HIV+ participants developed AIDS. Six (8.2\%) HIV+ participants had a detectable viral load during the study period in the form of low viral blips (51-130 copies/mL). Eleven (15\%) HIV+ participants had modifications to their cART during the study period, mostly representing cART optimization (e.g., tolerability; single dose to improve adherence). Of those, only 1 changed treatment and also had a detectable viral load during the study period.

In the FWM, a history of HAND was associated with reduced Cr at follow-up $(p<0.02$; Std $\beta=-0.26)$. In the PCC, a history of HAND was associated with reduced NAA at baseline $(p<0.02$; $\operatorname{std} \beta=0.19)$. There were no significant findings in relation to $\mathrm{Cr}$ and $\mathrm{mI}$ in the PCC. In the CA, HIV duration was associated with lower NAA at both baseline and follow-up $(p<0.004 ; \operatorname{Std} \beta=-0.26)$.

\section{Discussion}

To the best of our knowledge, covert neurochemical abnormalities in persons with stable HAND and to a lesser extent in cognitively normal HIV+ persons have not been described before. Indeed, when relating metabolite concentrations to the neurocognitive trajectories, different brain metabolic profiles emerged. Stable HAND was mainly characterized by PCC and FWM decreased cellular energy, whereas incident

Table 1 Summary of brain metabolite profile changes in the neurocognitive trajectory groups using the HIV- group as the statistical reference

\begin{tabular}{lllll}
\hline & FWM Cr & PCC Cr & PCC NAA & CA NAA \\
\hline Stable and cognitive intact & Not different, stable & Decreasing & Not different, stable & Not different, stable \\
\hline Stable HAND & Decreasing & Decreasing & Decreasing & Low, slightly decreasing \\
\hline Progressing HAND & Stably low & Decreasing & Low, sharply decreasing & Stably low \\
\hline Incident HAND & Sharply decreasing & Decreasing & Not different & Low, sharply increased \\
\hline
\end{tabular}

Abbreviations: CA NAA = caudate area N-Acetyl aspartate; FWM Cr = frontal white matter creatine; HAND = HIV-associated neurocognitive disorder; PCC Cr = posterior cingulate cortex creatine; PCC NAA = posterior cingulate cortex N-Acetyl aspartate.

a Results are indicative due to small sample size. 
Table 2 Differences in brain metabolite levels across the study period

\begin{tabular}{|c|c|c|c|c|c|c|c|c|c|c|c|c|c|c|}
\hline & \multicolumn{4}{|c|}{ BL } & \multicolumn{4}{|c|}{ FU } & \multirow[b]{3}{*}{ Group $p$} & \multirow[b]{3}{*}{ ES (Std $\beta)$} & \multirow[b]{3}{*}{ Time $p$} & \multirow[b]{3}{*}{ ES $(\operatorname{Std} \beta)$} & \multirow[b]{3}{*}{ Interaction $p$} & \multirow[b]{3}{*}{$E S(\operatorname{Std} \beta)$} \\
\hline & \multicolumn{2}{|c|}{ HIV- } & \multicolumn{2}{|c|}{ HIV+ } & \multicolumn{2}{|c|}{ HIV- } & \multicolumn{2}{|c|}{ HIV+ } & & & & & & \\
\hline & Mean & SD & Mean & SD & Mean & SD & Mean & SD & & & & & & \\
\hline \multicolumn{15}{|l|}{ FWM } \\
\hline NAA & 4.50 & 0.35 & 4.45 & 0.39 & 4.39 & 0.74 & 4.35 & 0.56 & 0.53 & 0.04 & 0.16 & 0.10 & 0.89 & 0.01 \\
\hline $\mathrm{Cr}$ & 3.07 & 0.34 & 2.98 & 0.33 & 3.12 & 0.38 & 2.91 & 0.33 & 0.0004 & 0.24 & 0.92 & -0.007 & 0.21 & 0.09 \\
\hline Cho & 2.40 & 0.42 & 2.37 & 0.34 & 2.40 & 0.34 & 2.44 & 0.40 & 0.89 & -0.01 & 0.54 & -0.05 & 0.53 & 0.05 \\
\hline $\mathrm{ml}$ & 1.09 & 0.28 & 1.14 & 0.25 & 1.14 & 0.20 & 1.13 & 0.25 & 0.50 & -0.05 & 0.59 & -0.04 & 0.35 & -0.07 \\
\hline Glx & 1.50 & 0.31 & 1.71 & 0.39 & 1.57 & 0.43 & 1.57 & 0.42 & 0.07 & -0.12 & 0.49 & 0.05 & 0.06 & -0.14 \\
\hline \multicolumn{15}{|l|}{ PCC } \\
\hline NAA & 5.24 & 0.54 & 5.11 & 0.61 & 5.21 & 0.62 & 4.81 & 1.04 & 0.02 & 0.16 & 0.15 & 0.10 & 0.21 & -0.09 \\
\hline $\mathrm{Cr}$ & 3.60 & 0.35 & 3.51 & 0.41 & 3.65 & 0.44 & 3.38 & 0.48 & 0.0046 & 0.19 & 0.55 & 0.04 & 0.15 & -0.10 \\
\hline Cho & 1.73 & 0.22 & 1.71 & 0.25 & 1.69 & 0.23 & 1.66 & 0.33 & 0.58 & 0.04 & 0.24 & 0.09 & 0.91 & -0.01 \\
\hline $\mathrm{ml}$ & 0.68 & 0.16 & 0.75 & 0.23 & 0.71 & 0.14 & 0.75 & 0.18 & 0.049 & -0.14 & 0.65 & -0.04 & 0.75 & -0.02 \\
\hline GIX & 2.14 & 0.43 & 2.11 & 0.47 & 1.91 & 0.40 & 1.87 & 0.46 & 0.78 & 0.02 & 0.0004 & 0.25 & 0.96 & -0.00 \\
\hline \multicolumn{15}{|l|}{$\mathrm{CA}$} \\
\hline NAA & 4.50 & 0.37 & 4.33 & 0.47 & 4.43 & 0.54 & 4.30 & 0.48 & 0.03 & 0.15 & 0.49 & 0.05 & 0.76 & 0.02 \\
\hline $\mathrm{Cr}$ & 3.39 & 0.42 & 3.32 & 0.35 & 3.32 & 0.53 & 3.21 & 0.60 & 0.29 & 0.07 & 0.19 & 0.09 & 0.77 & -0.02 \\
\hline Cho & 1.98 & 0.29 & 1.93 & 0.41 & 1.88 & 0.52 & 1.91 & 0.42 & 0.99 & -0.00 & 0.35 & 0.07 & 0.51 & 0.05 \\
\hline $\mathrm{ml}$ & 1.34 & 0.25 & 1.46 & 0.47 & 1.47 & 0.60 & 1.40 & 0.52 & 0.79 & -0.02 & 0.58 & -0.04 & 0.21 & -0.09 \\
\hline
\end{tabular}

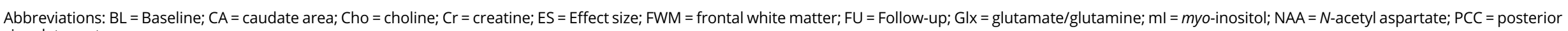
cingulate cortex 
HAND by steep cellular energy reduction particularly in the FWM and to a lesser extent in the PCC. Incident HAND was also characterized by a sharp increase in CA NAA, which we interpret as potential glial activation (see further on this below). Progressing HAND was characterized by sharp neuronal density reductions, particularly in the PCC and to a lesser extent in the CA. These effects were detected in people with both ANI and MND at baseline, although an MND baseline status yielded larger effect sizes. Of interest, stable intact participants were the closest to the HIV- controls' neurochemical profile, although as for other trajectory groups, their PCC Cr levels showed a longitudinal reduction.

The results partially confirm our hypothesis by demonstrating that neurochemical abnormalities are evolving, but principally in HIV+ persons with HAND regardless of whether it is stable, incident, or progressing, whereas those with normal cognition are relatively less affected. Stable HAND is the most common longitudinal trajectory in this cohort, and this has also been shown to be the case in other cART-treated cohorts internationally. ${ }^{4,17}$ Therefore, our findings have significant clinical ramifications by suggesting that persons with stable HAND (of whom the majority have $\mathrm{ANI}^{3}$; figure 1) are in fact experiencing slowly evolving neuropathogenesis whose nature remains to be elucidated. Of importance, our study offers new insights on the putative neuropathogenic processes.

Indeed, research into $\mathrm{Cr}$ depletion in the non-HIV population shows that part of the reduction may be systemic, possibly because of reduced uptake of $\mathrm{Cr}$ in the gut. ${ }^{18}$ Changes in the activity of the gut $\mathrm{Cr}$ transporter could perhaps be enhanced in chronic HIV infection because of increased microbial translocation and alterations to gut microbiota composition, ${ }^{19}$ whereas the latter has been associated with brain and cognitive dysfunctions. ${ }^{20,21}$ Decreased $\mathrm{Cr}$ levels may also arise because of alterations within brain tissue, as $\mathrm{Cr}$ is also synthesized within the brain. ${ }^{18} \mathrm{Cr}$ synthesis may occur variously in different cell types, with exchange of $\mathrm{Cr}$ and the precursor guanidine acetate occurring via the solute carrier 6A8 (SLC6A8). ${ }^{18} \mathrm{Cr}$ also leaks out of brain cells and into CSF via an as-yet-unidentified exchanger. Changes in the activity of this exchanger may, therefore, also lead to depletion.

Our study while smaller than the US HIV NeuroAIDS consortium study ${ }^{5}$ included an age-comparable control group. Using them as a longitudinal reference, we identified subgroups of HIV+ persons in whom HIV-related brain injury is slowly evolving or more rapidly progressing. This clinical grouping as opposed to a simple correlation between the cognitive level and metabolites concentration enhances the potential clinical utility of ${ }^{1} \mathrm{H}$-MRS and supports the clinical relevance of our neuropsychological methods for defining neurocognitive trajectories $^{22}$ (also used in the large longitudinal CHARTER study ${ }^{4,23}$ ). Furthermore, our findings support the neuropathogenic hypothesis that most of the brain damage in suppressed HIV infection is active, although mostly of a moderate magnitude. We showed this indirectly by demonstrating that legacy effects such as HIV duration and history of HAND only partially contribute to NAA abnormalities principally in the CA and to a lower extent in the PCC, as well as speeding up $\mathrm{Cr}$ reduction in the FWM. Based on our finding and that of others, ${ }^{11}$ we confirm that the use of $\mathrm{Cr}$ as a default reference should be discontinued and that the PCC should be routinely included to account for potential neurodegenerative changes. ${ }^{24}$

The finding of increased NAA in the CA for the incident HAND group (figure 2D) requires a more complex explanation. This "paradoxical" increase has been associated with microglia activation in a NeuroAIDS macaque model ${ }^{25}$ and potentially involves hypo-osmosis. ${ }^{26}$ Other non-HIV studies suggest that increased $\mathrm{Cr}$ can occur in the context of glutamate toxicity, ${ }^{18}$ although we did not detect a significant glutamate increase in this study, possibly because Glx is difficult to robustly resolve at $3 \mathrm{~T}^{11}$

A history of HAND modestly contributed to lower FWM Cr and lower PCC NAA, suggesting that HAND onset results in long-term CNS vulnerability. ${ }^{27}$ Such legacy effects on brain functions in chronic HIV infection are difficult to demonstrate, despite having been suspected to play a role for many years. ${ }^{28}$ Of importance, reductions in NAA and Cr levels in the PCC have been associated with abnormal aging and amyloid pathology in non-HIV populations ${ }^{24}$ - suggesting early neurodegeneration in the current sample. In this study, we detected HIV effects (including in the stable intact), which are the cause of concern, as this suggests that the chronic HIV+ population will potentially be at a greater risk of neurodegeneration as they age. ${ }^{29}$ Finally, the independent effect of HIV duration on NAA levels in the CA of HIV+ participants confirms and extends our previous finding in the cross-sectional baseline data. ${ }^{7}$ This provides further support for the notion that the CA is an early and specific target of HIV infection and cumulatively adds evidence that HIV duration is a risk factor for HIV-related brain injury in virally suppressed persons with a history of low nadir $\mathrm{CD} 4^{+} \mathrm{T}$-cell count $(<350$ copies $/ \mathrm{mL})$.

The study has several limitations. Although we enrolled both HIV- and HIV+ persons who were demographically comparable, the sample size was medium to large. Therefore, the total number of cases with clinically significant cognitive decline was relatively small, which affected our power, especially in incident HAND, but not the size of the effects, which were particularly medium to large in this subgroup. In addition, our study was composed of well-educated men who may be protected from the effects of HIV and aging, ${ }^{30}$ so the progression of neurochemical abnormalities in such a sample may have been somewhat diminished. Finally, the lost-to-follow-up cases had more frequent viral blips during the study period. Thus, the retained sample represented a particularly clinically stable group.

Our study has uncovered early evidence of different pathogenic processes associated with differential neurocognitive trajectories. Longer follow-up studies will be important to 
determine the prognosis of the slowly evolving neurochemical abnormalities and which factors may speed up progression (e.g., reaching age 60 years, chronic immune activation, immune senescence, and evidence of a neurodegenerative disease).

\section{Author contributions}

Conception and design of the study: L.A.C., B.J.B., and C.R. Acquisition and analysis of data: L.A.C., L.J., T.G., M.T., and K.M. Drafting a significant portion of the manuscript or figures: L.A.C., B.J.B., C.R., L.J., T.G., M.T., and K.M.

\section{Acknowledgment}

The authors thank all research participants without whom this research would not be possible. They also thank Bronwyn Overs for providing them with an improved batch version of the voxel white matter, gray matter, and CSF composition.

\section{Study funding}

This study was supported by the National Health and Medical Research Council of Australia project grant ID568746 (Cysique PI), the 2009-2012 postdoctoral Brain Science UNSW fellowship (Cysique), 2012 Merck Sharp Dome and Abbott support, the National Health and Medical Research Council of Australia Career Development Fellowship APP 1045400 (Cysique PI), and the Peter Duncan Neurosciences Unit (Head Prof. Bruce J. Brew). MSD and Abbott had no direct participation in the current study design, data analyses, and interpretation.

\section{Disclosure}

L.A. Cysique is on the editorial board of the Journal of AIDS o Clinical Research, Journal of Sexually Transmitted Diseases, World Journal of Neurology, Biomed Research International, and PLoS One and received research support from Gilead and NHMRC. L. Jugé received research support from The Rebecca L. Cooper Medical Research Foundation Ltd. T. Gates, M. Tobia, and K. Moffat report no disclosures. B.J. Brew served on the scientific advisory board of Biogen Idec, ViiV, and Merck Serono; received travel funding from Abbott and ViiV; serves on the editorial board of the International Journal of Tryptophan Research, Faculty of 1000, Journal of Neurovirology, and Neurobehavioral HIV Medicine; received publishing royalties from Oxford University Press and Cambridge University Press; consulted for GlaxoSmithKline, ViiV Healthcare, Boehringer Ingelheim, Abbott, Biogen Idec, and Novartis; and received research support from Eli Lilly, GlaxoSmithKline, Merck Serono, ViiV Healthcare, Biogen Idec, NIH NHMRC, NINDS, NIMH, University of New South Wales, University of Notre Dame-Sydney, and St. Vincent's Clinic Research Foundation. C. Rae received travel funding from Philips Healthcare; is an associate editor for Neurochemical Research; and received research support from the Australian Research Council. Go to Neurology.org/nn for full disclosure forms.
Received July 23, 2017. Accepted in final form October 10, 2017.

\section{References}

1. Cysique LA, Brew BJ. The effects of HIV and aging on brain functions: Proposing a research framework and update on last 3 years' findings. Curr Opin HIV AIDS 2014; 9:355-364.

2. Masters MC, Ances BM. Role of neuroimaging in HIV-associated neurocognitive disorders. Semin Neurol 2014;34:89-102.

3. Gott C, Gates T, Dermody N, Brew BJ, Cysique LA. Cognitive change trajectories in virally suppressed HIV-infected individuals indicate high prevalence of disease activity. PLoS One 2017;12:e0171887.

4. Heaton RK, Franklin DRJr, Deutsch R, et al. Neurocognitive change in the era of HIV combination antiretroviral therapy: the longitudinal CHARTER study. Clin Infect Dis 2015;60:473-480.

5. Gongvatana A, Harezlak J, Buchthal S, et al. Progressive cerebral injury in the setting of chronic HIV infection and antiretroviral therapy. J Neurovirol 2013;19:209-218.

6. Kanapathipillai R, McManus H, Kamarulzaman A, et al. The significance of HIV "blips" in resource-limited settings: Is it the same? Analysis of the Treat Asia HIV Observational Database (TAHOD) and the Australian HIV Observational Database (AHOD). PLoS One 2014;9:e86122.

7. Cysique LA, Moffat K, Moore DM, et al. HIV, vascular and aging injuries in the brain of clinically stable HIV-infected adults: A (1)H MRS study. PLoS One 2013;8: e61738.

8. Cysique LA, Heaton RK, Kamminga J, et al. HIV-associated neurocognitive disorder in Australia: A case of a high-functioning and optimally treated cohort and implications for international neuroHIV research. J Neurovirol 2014;20:258-268.

9. Kanowski M, Kaufmann J, Braun J, Bernarding J, Tempelmann C. Quantitation of simulated short echo time $1 \mathrm{H}$ human brain spectra by LCModel and AMARES. Magn Reson Med 2004;51:904-912.

10. Singh S, Mistry S, Jefferson S, et al. A magnetic resonance spectroscopy study of brain glutamate in a model of plasticity in human pharyngeal motor cortex. Gastroenterology 2009; 136:417-424.

11. Rae CD. A guide to the metabolic pathways and function of metabolites observed in human brain 1H magnetic resonance spectra. Neurochem Res 2014;39:1-36.

12. Partial volume code for Philips MRS data licensed under a Creative commons AttributionShareAlike 3.0 Unported License. [computer program]. Available at: http://biu.bangor.ac. $\mathrm{uk} /$ projects.php.en?menu=2\&catid=10152\&subid=0. Accessed May 2017.

13. Lane TA, Moore DM, Batchelor J, Brew BJ, Cysique LA. Facial emotional processing in HIV infection: Relation to neurocognitive and neuropsychiatric status. Neuropsychology 2012;26:713-722.

14. Carey CL, Woods SP, Gonzalez R, et al. Predictive validity of global deficit scores in detecting neuropsychological impairment in HIV infection. J Clin Exp Neuropsychol 2004;26:307-319.

15. Blackstone K, Moore DJ, Franklin DR, et al. Defining neurocognitive impairment in HIV: Deficit scores versus clinical ratings. Clin Neuropsychol 2012;26:894-908.

16. Letendre S. Central nervous system complications in HIV disease: HIV-associated neurocognitive disorder. Top Antivir Med 2011;19:137-142.

17. Sacktor N, Skolasky RL, Seaberg E, et al. Prevalence of HIV-associated neurocognitive disorders in the multicenter AIDS cohort study. Neurology 2016;86:334-340.

18. Rae CD, Broer S. Creatine as a booster for human brain function. How might it work? Neurochem Int 2015;89:249-259.

19. Williams B, Landay A, Presti RM. Microbiome alterations in HIV infection a review. Cell Microbiol 2016;18:645-651.

20. Hoenigl M, de Oliveira MF, Perez-Santiago J, et al. (1->3)-beta-D-Glucan levels correlate with neurocognitive functioning in HIV-infected persons on suppressive antiretroviral therapy: A cohort study. Medicine (Baltimore) 2016;95:e3162.

21. Vera JH, Guo Q, Cole JH, et al. Neuroinflammation in treated HIV-positive individuals: A TSPO PET study. Neurology 2016;86:1425-1432.

22. Cysique LA, Franklin D Jr, Abramson I, et al. Normative data and validation of a regression based summary score for assessing meaningful neuropsychological change. J Clin Exp Neuropsychol 2011;33:505-522.

23. Cysique LA. Should longitudinal multisite studies become the new standard for investigating neurocognitive functions in HIV infection? Clin Infect Dis 2015;60:481-482.

24. Murray ME, Przybelski SA, Lesnick TG, et al. Early Alzheimer's disease neuropathology detected by proton MR spectroscopy. J Neurosci 2014;34:16247-16255.

25. Ratai EM, Annamalai L, Burdo T, et al. Brain creatine elevation and $\mathrm{N}$-acetylaspartate reduction indicates neuronal dysfunction in the setting of enhanced glial energy metabolism in a macaque model of neuroAIDS. Magn Reson Med 2011;66:625-634

26. Bothwell JH, Rae C, Dixon RM, Styles P, Bhakoo KK. Hypo-osmotic swellingactivated release of organic osmolytes in brain slices: implications for brain oedema in vivo. J Neurochem 2001;77:1632-1640.

27. Young AC, Yiannoutsos CT, Hegde M, et al. Cerebral metabolite changes prior to and after antiretroviral therapy in primary HIV infection. Neurology 2014;83:1592-1600.

28. Brew BJ. Evidence for a change in AIDS dementia complex in the era of highly active antiretroviral therapy and the possibility of new forms of AIDS dementia complex. AIDS 2004;18(suppl 1):S75-S78.

29. Cysique LA, Hewitt T, Croitoru-Lamoury J, et al. APOE epsilon4 moderates abnormal CSF-abeta-42 levels, while neurocognitive impairment is associated with abnormal CSF tau levels in HIV+ individuals - a cross-sectional observational study. BMC Neurol 2015;15:51.

30. Cysique LA, Brew BJ. Prevalence of non-confounded HIV-associated neurocognitive impairment in the context of plasma HIV RNA suppression. J Neurovirol 2011;17:176-183. 


\section{Neurology \\ Neuroimmunology \& Neuroinflammation}

\section{Covertly active and progressing neurochemical abnormalities in suppressed HIV infection \\ Lucette A. Cysique, Lauriane Jugé, Thomas Gates, et al. \\ Neurol Neuroimmunol Neuroinflamm 2018;5; \\ DOI 10.1212/NXI.0000000000000430}

This information is current as of January 3, 2018

\section{Updated Information \& Services}

References

Citations

Subspecialty Collections

Permissions \& Licensing

Reprints including high resolution figures, can be found at: http://nn.neurology.org/content/5/1/e430.full.html

This article cites 29 articles, 1 of which you can access for free at: http://nn.neurology.org/content/5/1/e430.full.html\#\#ref-list-1

This article has been cited by 2 HighWire-hosted articles: http://nn.neurology.org/content/5/1/e430.full.html\#\#otherarticles

This article, along with others on similar topics, appears in the following collection(s):

HIV dementia

http://nn.neurology.org//cgi/collection/hiv_dementia

MRS

http://nn.neurology.org//cgi/collection/mrs

Information about reproducing this article in parts (figures,tables) or in its entirety can be found online at:

http://nn.neurology.org/misc/about.xhtml\#permissions

Information about ordering reprints can be found online:

http://nn.neurology.org/misc/addir.xhtml\#reprintsus

Neurol Neuroimmunol Neuroinflamm is an official journal of the American Academy of Neurology.

Published since April 2014, it is an open-access, online-only, continuous publication journal. Copyright

Copyright $\odot 2018$ The Author(s). Published by Wolters Kluwer Health, Inc. on behalf of the American

Academy of Neurology.. All rights reserved. Online ISSN: 2332-7812.

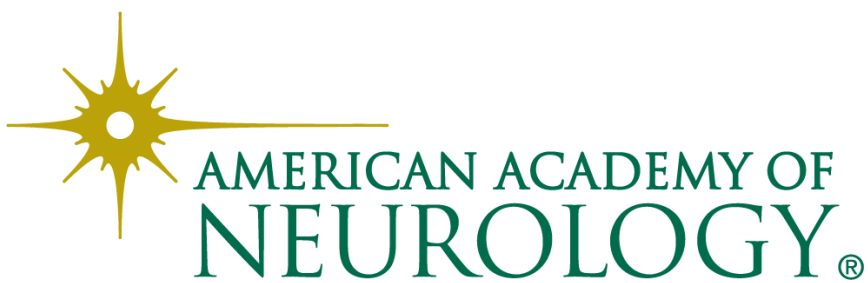

